\title{
Achieving Health Equity through Health Care Reform: Current State and Outlook
}

Ifeoma A. Inneh ${ }^{1,2,3 *}$

${ }^{1}$ Department of Orthopaedic Surgery, Baylor College of Medicine/Texas Children's Hospital, Houston TX, USA

${ }^{2}$ Division of Public Health and Policy, Faculty of Health and Life Sciences, School of Medicine, University of Liverpool, Liverpool, UK

${ }^{3}$ Department of Occupational and Industrial Orthopedic, New York University, USA

\begin{abstract}
Despite advances in medicine and health care reform in the United States (US), inequities and disparities whether in health status and/or care persist(s) for certain vulnerable or disadvantaged groups. This paper presents a perspective on the current state of inequities and disparities in US health care system as it relates to certain initiatives in an era of health care reform, and possible recommendations to further improve the outlook of achieving health equity
\end{abstract}

Keywords: Health equity; Healthcare reform; Health disparities; Health inequities; Health equity and health reform

\section{Introduction}

Health, the "state of complete physical, mental, and social wellbeing, and not merely the absence of disease or infirmity," is a fundamental human right without distinction of race, political belief, economic or social condition." As such, health and access to care to maintain the highest level of health should be equitable $[1,2]$. Despite advances in medicine and health care, inequities and disparities whether in health status and/or care persist for certain vulnerable or disadvantaged groups [3]. Though often used interchangeably, health inequities are the "differences in health that are avoidable, unfair, and unjust, often influenced by social, economic, and environmental conditions," while health disparities are the "differences in health outcomes among groups of people" [2]. These definitions suggest that many of the contributing factors to inequitable health, are often external to and beyond an individual's direct control. Specific to health care, "equitable care" would mean individualized care that is catered to the specific needs of a particular individual, in order to optimize his/her health outcomes. In the United States (US), despite high health care costs and technological advances, its health care system still exhibits wide inequities and disparities in health and health care; prompting the need for better understanding of primary drivers to inform policies. Over the past decades, the US government has set goals aimed at eliminating health disparities [4]. This brought about health care reform, also known as the Patient Protection Affordable Care Act (ACA), enacted in 2010 with the aim to "make affordable health insurance available to more people, thereby improving access and quality [5]." Its passage helped decrease the gap between insured and uninsured/underinsured particularly for economically disadvantaged groups, and is anticipated to lower costs [5]. Although, data from the initial years after the law's implementation show large reductions in the rates of uninsurance/underinsurance among low-income groups [6-13], significant inequities and disparities in health remain [14-16]. Under this law, several other initiatives such as new payment models, more streamlined quality metrics and population health, have been introduced. Given that the impact of coverage expansion has been comprehensively addressed in other literature, the purpose of this paper is to present a concise perspective on the current state of inequities and disparities in the US health care system as it relates to these other initiatives in an era of health care reform, and present possible recommendations to further improve the outlook of achieving health equity. To ensure a comprehensive analysis of the topic, both terms- inequities and disparities- are applied in the context of this paper [16].

\section{Payment Reform: Introduction of Alternative Payment Models}

Payment reform is an important component of health care reform given the escalating costs of health care. It focuses on the shift away from paying for "volume" to "value" with the ultimate aim to deliver safe, timely, patient-centered, effective, efficient, equitable and affordable care [17]. From another perspective, it also aims to decrease fraud and abuse. At the federal government level, alternative payment models that move away from traditional fee-for-service model to a value-based model such as single episode bundled payments and population-based accountable care organization (ACO) models with focus on improved quality and reduced cost are rapidly becoming the norm. These new models involve transforming health care delivery, demanding that care providers (i.e. clinicians, hospitals, health systems, etc.) meet certain quality thresholds while containing costs. They also encourage using care teams and information technology to improve efficiency and health outcomes. Commercial insurers in the private sector are also following suit. With regards to containing costs, the results are inconclusive [18-22]. Specific to addressing inequities in access to care, state level Medicaid programs such as the Delivery System Reform Incentive Payment program and other section 1115 waivers, aims to restructure health care delivery particularly for low-income patients [23].

Specific to health equity, while improved insurance coverage and targeted efforts by some care providers (health care systems) have narrowed some disparities, significant gaps remain particularly for certain minority groups even with the same insurance and socioeconomic status $[14,24]$. This suggests that despite the intended aim of alternative payment models in improving value of care, they generally may not be directly encouraging or supporting the reduction of health inequities and disparities. As such, any new initiatives or

*Corresponding author: Ifeoma A. Inneh, Department of Occupational and Industrial Orthopedic, New York University, USA, Tel: 1.469.708.8184; E-mail: ifyineh@hotmail.com

Received August 05, 2018; Accepted November 14, 2018; Published November 21,2018

Citation: Inneh IA (2018) Achieving Health Equity through Health Care Reform Current State and Outlook. Health Care Current Reviews 6: 233. doi: 10.4172/2375 4273.1000233

Copyright: (C) 2018 Inneh IA. This is an open-access article distributed under the terms of the Creative Commons Attribution License, which permits unrestricted use, distribution, and reproduction in any medium, provided the original author and source are credited. 
demonstrations calling for alternative payment models ought to strategically link inequities and disparities reduction to payment. In an article published by DeMeester et al., it was reported that applicants who responded to the Finding Answers: Solving Disparities through Payment and Delivery System Reform program sponsored by the Robert Wood Johnson Foundation, generally did not link payment reform tightly to disparities reduction [25]. Perhaps this challenge is attributable to the ambiguity in linking and/or measuring inequities and disparities as it relates to value-based care. The bottom line is that while current alternative payment models theoretically have the potential to help bridge the equity gap as it relates to access, they are still fairly premature to ascertain ultimate ability to fully address inequities and disparities.

\section{Quality Metrics and Measures}

Though quality metrics are strongly linked to alternative payment models above, for the purpose of this paper, they are discussed separately. Measuring the quality of health care is a necessary step in health care quality improvement [26,27]. It helps "prevent the overuse, underuse, and misuse of health care services to ensure patient safety; identify what works or doesn't in health care to drive improvement; hold health insurance plans and care providers accountable for providing high-quality care; measure and address disparities in how care is delivered and in health outcomes; as well as guide consumers in making informed choices about their care [28]." Quality measures form the metrics with which health care quality is assessed across the full continuum of health care delivery. They can be assessed in four domains: structure (capacity, systems, and processes), process (maintain or improve health, either for healthy people or for those diagnosed with a health care condition), or outcome (impact of the health care service or intervention on the health status of patients) [29].

The enactment of the ACA prompted the initiation of several value-based quality incentive programs for inpatient and outpatient care settings such as the Hospital Readmissions Reduction, Hospital Value Based Purchasing, End-Stage Renal Disease Quality Incentive, Physician Value-Based Modifier, Hospital Acquired Conditions, Skilled Nursing Facility Value Based, Home Health Value Based and Merit-Based Incentive Payment System, Ambulatory Surgical Center Quality Reporting, Hospital Outpatient Quality Reporting, Hospital Inpatient Quality Reporting, Physician Quality Reporting System, and Inpatient Rehabilitation Facility Quality programs, to mention a few [30]. Each program comprises of metrics necessary to meet priorities for improved value- health care outcomes, quality, safety, efficiency and satisfaction- for patients, all of which are tied to reimbursement. While there may have been decline in some quality related outcomes such as hospital acquired conditions and readmissions in recent years, disparities still exist in their distribution among certain vulnerable, disadvantaged and/or minority groups for certain medical conditions [14,31-34].

This is perhaps attributable to the fact that there has been no notable implementation of "measures/metrics" for quality performance as it relates to health equity. In fact, the Institute of Medicine's seminal report "Crossing the Quality Chasm [35]" identified health equity as a key domain of quality. For many years, stakeholders (i.e. care providers, professional organizations, health services researchers and policy makers) have recommended stratifying clinical performance measures by sociodemographic factors such as race, ethnicity, and socioeconomic status to identify disparities and motivate change to improve equity and quality [21,36]. The National Quality Forum developed a four-part strategy guide to demonstrate how measurement and associated policies can contribute to eliminating disparities and promote health equity. They include identification and prioritization of areas to reduce health disparities, implementation of evidence-based interventions to reduce disparities, investment in development and use of health equity performance measures, and financial incentives for the reduction of health disparities and achievement of health equity [37]. However, this is still yet to fully come to fruition.

\section{Population Health}

As previously stated, inequities and disparities in health are shaped by multilevel socioecological influences sometimes outside of an individual's direct control. They include health care and individual behavior, which in turn are shaped by the physical, political, social and economic environment, collectively defined as "social determinants of health [38]." These social determinants of health are complexly interrelated, making simplistic explanations of the causes of health inequities and disparities difficult [6]. With increasing efforts to reduce health spending and improve health outcomes, federal and state governments, as well as the private sector, are testing ways in which to address these social determinants of health. The goal is to break down silos in the system to overcome the challenge of paying for these efforts. One of such avenues is through population health. Population health is defined as "the health outcomes of a group of individuals, including the distribution of such outcomes within the group [39]." A more comprehensive definition would include "the distribution and determinants of health outcomes, as well as facilitation of care delivery across/among groups of individuals in order to improve their health outcomes, and lower costs [40-44].” It advocates wellness, prevention and chronic disease management through avenues like medical homes, particularly for those who face social barriers to health, and is paramount to the reform of the care delivery system [45]. It is a combination of integrated healthcare, public health and health policy. As such, requires initiatives that involve collaboration across various sectors like public health, social services and public policy.

As population health becomes more of a focus of health care, providers are realizing that the challenges in operationalizing population health initiatives, identifying which metrics/measures to track progress, prevail [46]. One of the primary drivers of such challenges involves "data." It is becoming more evident that data outside of primary clinical diagnosis can provide a broader perspective on potential drivers of a patient's health status, as well as help identify approaches to improving the effectiveness of care $[47,48]$. However, there is no standardization for the definition, capture and/ or representation of data on social determinants of health in the care process $[47,49]$. Other challenges include the inability to establish a "cause-and-effect" relationship between these social determinants of health and health outcomes. The current evidence suggests that though it may be premature to ascertain the role of population health in achieving health equity, the theoretical framework does suggest that it has the potential to help mitigate inequities and disparities in health and health care. This can only be proven with time.

\section{Policy Implications and Recommendations}

Given the rapid increase of new care delivery initiatives through health care reform, it is imperative to advance understanding of how they might be better designed and implemented to bridge the equity gap, as this is simply the right thing to do. However, doing so must be feasible and sustainable. To ensure that health equity is central in ongoing health care reform efforts and to drive meaningful improvement, the health care system need(s) strategy (ies) that 
include(s) metrics sensitive to addressing inequities and disparities in health and health care. This will involve the use of evidence-based approaches to identify and address root causes of these inequities and disparities. Specific recommendations are outlined as follows, and are made with the acknowledgement that they may not be straightforward and/or easy.

- Create and/or improve financial incentives in payment reform to support intrinsic motivation of care providers and payers to achieve equity. This would include introduction of more rigorous evidence-based demonstration programs that integrate a "disparities/inequities-reduction" component based on social determinants of health, in care innovation. This could further guide the introduction of more risk-based models that fairly compensate care providers who must cater to certain vulnerable patient populations. This is based on the premise that while value-based payment initiatives have the potential to improve quality of care, there is concern that they may also lead to the widening of health disparities if providers with limited resources are unable to respond effectively to incentives $[32,50,51]$.

- Ensure population health interventions are embedded in a systematic manner and are culturally "tailored" for the specific settings and populations in which care providers make achieving health equity a high priority. This could include pilot or demonstration programs to test what works in unique situations.

- Standardize and incentivize the efficient collection of data on social determinants of health as a standard of care and be part of a patient's health record. Cantor et al. recommend collection of such data through financial or quality measures, and expanding the body of research that measures the impact of acting on the information collected. They posit that this "offers tremendous potential for improved care and health, including a better understanding of the influence of neighborhood characteristics on health, and improved connections between providers of medical care and community services [47]."

- Health care institutions prioritize health equity as part of their institutional culture. It is well known that multiple factors cause disparities in health outcomes, including unconscious bias and cultural insensitivity by care providers [52]. Therefore, awareness in the form of trainings/education on health equity and institution-wide culture change are necessary. Other ways include encouraging a more diverse workforce of health care workers across various disciplines, roles and levels of the institution; making education of inequities and disparities part of an institution's mission and vision with clearer indications as to how they will be achieved; as well as investment in the data infrastructure necessary for stratifying performance measures [53].

- Engage target populations (i.e. individuals and communitiesboth social and political-) who will be most directly affected by health care reform, and institutions across various sectors early on in future initiatives. Important avenues include the role of Patient Centered Outcomes Research Institute's (PCORI) pipeline to proposal initiative that encourage public-private partnerships between patients, clinicians, other front-line caregivers, and others across the healthcare community to participate in high-quality patient-centered outcomes research
[54]. Since its formation approximately 7-8 years ago, PCORI has been instrumental in fostering patient-centered and comparative effectiveness research with billions of dollars for various health conditions-particularly those of high cost and impact-. Another example includes the Centers for Disease Control and Prevention's embrace of the Health in All Policies (HiAP) initiative, which could yield potential promises in addressing health and care inequities to achieve health equity [55,56]. Given that health is created largely outside of the health care sector, these relationships are critical to addressing these problems and long term sustainability of any fruitful strategies. Marusic et al. state that while inclusive, consultative policy processes offer no guarantee against conflict, they may pay dividends over time $[57,58]$.

- As a follow up to the preceding point, increase funding for more "pragmatic" research around social determinants of health to study how they contribute to ongoing health inequities and disparities that exist at disproportionate rates across or among certain gender, racial, ethnic, geographical and/or economic groups. These research should allow for exploration of causal relationships between social determinants of heath and health outcomes, develop better metrics/measures for quality performance on equity, as well as the application of evidencebased strategies to inform better, and timely evaluation of existing interventions for population health management. As recommended through a panel review by Sampson et al. future research should also "embrace broad and inclusive themes at the individual and population levels; as well as encourage development of unique transdisciplinary training programs to build research capacity [4]."

\section{Conclusion}

Achieving health equity is a vital aim of global public health efforts. Any counter pressures to pull back on efforts to address inequities and disparities in health and health care have implications for human capital and the economy. This is particularly important to note in the current presidential administration, given that the issue of health care is a priority for citizens and residents of the US.

In summary, while certain aspects of US health care reform offer pathway(s) to achieving health equity, the complex interplay of health, social and political factors could impede its success. To ensure that the strides made in reforming the US health system (includes ongoing efforts to eliminate inequities and disparities) are sustainable in the long-term, political differences must be put aside at all branches of government to work towards the common good of the people and ensure that necessary resources are provided from the viewpoint of an investment estimated to yield positive returns in the future.

\section{References}

1. Constitution of the world health organization

2. healthypeople.gov/2020/about/foundation-health-measures/Disparities

3. Anderson AC, O'Rouke E, Chin MH, Ponce NA, Bernheim SM, et al. (2018) Promoting health equity and eliminating disparities through performance measurement and payment reform. Health Affairs 37: 371-377.

4. Samspon (2016) Reducing health inequities in the U.S: Recommendations from the NHLBl's health inequities think tank meeting. J Am Coll Cardiol 68: 517-524.

\section{5. https://www.healthcare.gov/glossary/affordable-care-act/}

6. Benitez JA, Creel L, Jennings J (2016) Kentucky's medicaid expansion showing early promise on coverage and access to care. Health Affairs 35: 528-354. 
7. Levy J (2008) In US uninsured rate lowest since. Gallup well-being.

8. Carman KG, Eibner C (2014) Changes in health insurance enrollment since 2013: Evidence from the RAND health reform opinion study. Rand Health $Q$ 4: $1-8$

9. Collins SR, Rasmussen PW, Doty MM (2014) Gaining ground: Americans' health insurance coverage and access to care after the affordable care act's first open enrollment period. New York: Commonwealth Fund.

10. Long SK, Kenney GM, Zuckerman S (2014) Quick take: Number of uninsured adults falls by 5.4 Million since 2013 .

11. Levy J (2013) US Uninsured Rate Drops to 13.4\%. Gallup Well-Being.

12. Clemans-Cope L, Karpman M, Weiss A, Anderson N (2014) Increase in medicaid under the aca reduces uninsurance, According to Early Estimates.

13. Sommers BD, Blendon RJ, Orav EJ, Epstein AM (2016) Changes in utilization and health among low-income adults after medicaid expansion or expanded private insurance. JAMA Intern Med 176: 1501-1509.

14. National healthcare quality and disparities report 2016.

15. Yue D, Rasmussen PW, Ponce NA (2018) Racial/ethnic differential effects of medicaid expansion on healthcare access. Health Serv Res 53: 3640-3656.

16. Gaffney A, McCormick D (2017) The affordable care act: Implications for health-equity. Lancet 389: 1442-52.

17. Bentacourt JR, Tan-McGrory A, Kenst KS, Phan TH, Lopez H (2017) Organizational change management for health equity: Perspectives from the disparities leadership program. Health Affairs 36: 1095-1101.

18. Iorio R, Clair AJ, Inneh IA, Slover JD, Bosco JA, et al. (2016) Early results of medicare's bundled payment initiative for a 90-day total joint replacement episode of care. Journal of Arthroplasty 343-350

19. McWilliams M, Hatfield LA, Chernew M, Landon BE, Schwartz AL (2016) Early performance of accountable care organizations in Medicare. NEJM Catalys 374: $2357-2366$

20. Sen AP, Chen LM, Samson LW, Epstein AM, Joynt Maddox KE (2018) Performance in the Medicare shared savings program by accountable care organizations disproportionately serving dual and disabled populations. Med Care 56: 805-811.

21. Courtney PM, Bohl DD, Lau EC, Ong KL, Jacobs JJ, et al. (2018) Risk adjustment is necessary in Medicare bundled payment models for total hip and knee arthroplasty. J Arthroplasty 33: 2368-2375.

22. Salzberg CA, Bitton A, Lipsitz SR, Franz C, Shaykevich S, et al. (2017) The impact of alternative payment in chronically III and older patients in the patientcentered medical home. Med Care 55: 483-492.

23. Driving health care innovation through DSRIP: State of the States.

24. Bentacourt JR (2014) In pursuit of high-value healthcare: The Case for improving quality and achieving equity in a time of healthcare transformation. Front Health Serv Manage 30: 16-31.

25. DeMeester RH, Xu LJ, Nocon RS, Cook SC, Ducas AM, et al. (2017) Solving disparities through payment and delivery system reform: A program to achieve health equity. Health Affairs 36: 1133-1139.

26. Vincent C, Burnett S, Carthey J (2014) Safety measurement and monitoring in healthcare: A framework to guide clinical teams and healthcare organisations in maintaining safety. BMJ Qual Saf 23: 670-677.

27. US department of health and human services health resources and services administration. "Quality Improvement".

28. Measuring healthcare quality: An overview of quality measures.

29. Agency for Healthcare quality and research (2018) Types of quality measures.

30. What are value-based programs 2018

31. Okike K, Chan PH, Prentice HA, Paxton EW, Navarro RA (2018) Association between race and ethnicity and hip fracture outcomes in a universally insured population. J Bone Joint Surg Am 100: 1126-1131.
32. Figueroa JF, Zheng J, Orav JE, Epstein AM, Jha AK (2018) Medicare program associated with narrowing hospital readmission disparities between black and white patients. Health Affairs 37: 654-661.

33. Romero T, Greenwood KL, Glaser D (2018) sex differences in acute myocardia infarction hospital management and outcomes: Update from facilities with comparable standards of quality care. J Cardiovasc Nurs 568-575.

34. Chen HF, Nevola A, Bird TM, Karim SA, Morris ME, et al. (2018) Understanding factors associated with readmission disparities among Delta region, Delta state, and other hospitals. Am J Manag Care 24: e150-e156.

35. Crossing the quality chasm: A new health system for the 21 st century.

36. Inneh IA (2015) The combined influence of sociodemographic, preoperative comorbid and intraoperative factors on longer length of stay and readmissions after elective primary total knee arthroplasty. J Arthroplasty 30: 1883-1886.

37. National Quality Forum (2017) A Roadmap for promoting health equity and eliminating disparities: The four i's for health equity.

38. Social determinants of health.

39. Kindig D, Stoddart G (2003) What is population health? Am J Public Health 93: $380-383$.

40. Tech Target Search Health IT (2016) Population health management.

41. Frieden TR (2010) A framework for public health action: The health impact pyramid. Am J Public Health 100: 590-595.

42. "National prevention strategy" 2011.

43. Transforming healthcare: From episodic interactions to population health management.

44. Harwood JL, Butler CA, Page AE (2016) Patient-centered care and population health: Establishing their role in the orthopaedic practice. J Bone Joint Surg Am 98: e40.

45. Agency for healthcare quality and research. "Defining the PCMH.

46. Crawford M, McGinnis T (2015) Population health in medicaid delivery system reforms. The reforming states group. Millbank Memorial Fund.

47. Cantor $M$, Thorpe $L$ (2018) Integrating data on social determinants of health into electronic health records. Health Affairs 37: 585-590.

48. Daniel H, Bornstein SS, Kane GC (2018) Addressing Social determinants to improve patient care and promote health equity: An American college of physicians position paper. Ann Intern Med 168: 577-593.

49. Capturing social and behavioral domains and measures in electronic health records.

50. Jha AK, Orav EJ, Epstein AM (2010) The effect of financial incentives on hospitals that serve poor patients. Ann Int Med 153: 299-306

51. Ryan AM, Blustein J, Casalino LP (2012) Medicare's flagship test of payfor-performance did not spur more rapid quality improvement among lowperforming hospitals. Health Affairs 31: 797-805

52. Smedley BD, Stith AY, Nelson AR (2003) Unequal treatment: Confronting racia and ethnic disparities in Healthcare.

53. Chin MH (2017) Movement advocacy personal relationships and ending healthcare disparities. J Natl Med Ass 109: 33-35.

54. Patient centered outcomes research institute (PCORI). "Pipeline to proposa initiative."

55. Centers for disease control and prevention (2018) "Health in all policy."

56. Hall RL, Jacobson PD (2018) Examining whether the health-in-all-policies approach promotes health equity. Health Affairs 37: 364-370.

57. Marusic D, Rupel VP (2016) Health care reforms. Zdr Varst 55: 225-227.

58. De Leeuw E (2017) Engagement of sectors other than health in integrated health governance, policy, and action. Annu Rev Public Health 38: 329-349. 